\title{
ENTOURAGES, DENSITY, CAUCHY MAPS, AND COMPLETION
}

\author{
JORGE PICADO AND ALEŠ PULTR
}

\begin{abstract}
We study uniformities and quasi-uniformities (uniformities without the symmetry axiom) in the common language of entourages. The techniques developed allow for a general theory in which uniformities are the symmetric part. In particular, we have a natural notion of Cauchy map independent of symmetry and a very simple general completion procedure (perhaps more transparent and simpler than the usual symmetric one).
\end{abstract}

\section{INTRODUCTION}

For the description of (symmetric) uniformity in the point-free context one typically uses the geometrically very natural Tukey type approach of a specific system of covers. For the non-symmetric case (although also here an approach using a specific system of pairs of covers is possible), it is natural to adopt an alternative definition using entourages (modelling the system of neighborhoods of the diagonal in the product $X \times X$ from the classical Weyl definition). It is not immediately clear that the two approaches are equivalent: the point-free (localic) products are not conservative, that is, the square $L \times L$ of locales does not quite correspond to the square $X \times X$ of topological spaces (see e.g. $[15,24])$. But they are $([20,21])$. This fact is somewhat surprising; it is deeper than the classical equivalence, and can be used to advantage (see e.g. the solution of an open problem in [23]).

As the title suggests, one of the topics of our paper is the concept of entourages. It is an ideal background for treating uniformities in (not necessary symmetric) generality (with the symmetric uniformity as a - simpler - special case). It provides an expedient common language enabling us, in particular, to present a natural theory of completeness not dependent on symmetry. We discuss this technique in more detail

Date: August 19, 2018.

2010 Mathematics Subject Classification. 06D22, 18B30, 54E15, 54D35.

Key words and phrases. Frame, locale, sublocale, uniform frame, quasi-uniform frame, entourage, uniform map, uniform dense embedding, Cauchy map, complete, completion. 
than necessary just for this purpose, believing that some of its aspects are interesting in themselves.

Density of uniform sublocales is crucial for understanding completeness. It also has some features that have not been discussed in the literature (in particular, in the non-symmetric case, with entourages). Therefore we give it a somewhat more extensive treatment.

As for the completeness questions, the reader is probably aware of a rich literature concerning this topic. To name just a few: there is the pioneering Isbell paper [14], then Kř́iž [17], Banaschewski [2, 3], Banaschewski and Pultr [6, 7, 8], Pultr and Tozzi [25], Banaschewski, Hong and Pultr [4], Hong and Kim [13]. Extending the topic to the non-symmetric case opened new vistas and brought more understanding to some of the aspects (Frith and Hunsaker [11], Frith, Hunsaker and Walters-Wayland [12], Kim [16]). The perspectives differ: there is Isbell's theory of feet, another one using the idea of Samuel compactification, yet another focusing on the behavior of uniform dense embeddings; in the non-symmetric case one uses the technique of biframes. Our approach is closest to that of the uniform dense embeddings, but the perspective is different. The central point here is the notion of Cauchy map. The natural construction of a largest frame in which a uniform frame is still densely embedded has a feature which has so far not been exploited, it provides a universal Cauchy map. Focusing on this we obtain a simple and more transparent completion procedure. Moreover, universality does not dependent on symmetry so that this approach to completion automatically yields the desired general result (with the symmetric one as a special case).

The paper is organized as follows. After Preliminaries we concentrate in Section 2 on the technique of entourages. This is then used throughout the rest of the paper. In particular it is consequently applied in Section 3 for introducing uniformities and quasi-uniformities and their basic properties. Next, in Section 4 we discuss density in uniform and quasi-uniform contexts; as in the whole of the paper we use, whenever it is of advantage, the localic technique. Section 5 is devoted to the pivot of our approach to completeness, namely to Cauchy maps (it should be noted that it was B. Banaschewski who proposed to view Cauchy maps as generalized Cauchy points, see e.g. [3]). It turns out that the construction used so far to produce a minimal dense superlocale in fact produces a universal Cauchy map (the proof of this is very easy, easier than the original minimal density one, probably because the Cauchy context is somewhat more natural). Then, in Section 
6, we obtain what we want to have about completeness and completion as simple applications. In Section 7 we add a few remarks on the non-symmetric case and its relation with the symmetric one.

\section{Preliminaries}

1.1. Frames and locales. Recall that a frame is a complete lattice $L$ satisfying the distributive law

$$
a \wedge(\bigvee B)=\bigvee\{a \wedge b \mid b \in B\}
$$

for all $a \in L$ and all subsets $B \subseteq L$. A frame homomorphism $h: L \rightarrow M$ preserves all joins (including the void one, the bottom 0 ) and all $f$ nite meets (including the top 1 ). The resulting category will be denoted by Frm. A typical frame is the lattice $\Omega(X)$ of all open sets of a topological space $X$; if $f: X \rightarrow Y$ is a continuous map then $\Omega(f)=\left(U \mapsto f^{-1}[U]\right): \Omega(Y) \rightarrow \Omega(X)$ is a frame homomorphism. Thus one has a contravariant functor $\Omega$ : Top $\rightarrow$ Frm (where Top is the category of topological spaces). Setting $\mathbf{L o c}=\mathbf{F r m}^{\mathrm{op}}$ one obtains the category of locales. Then $\Omega$ becomes a covariant functor Top $\rightarrow$ Loc; furthermore, restricted to the subcategory of sober spaces it is a full embedding. Thus one can think of locales as a generalization of (sober) topological spaces.

1.1.1. It is of advantage to view Loc as a concrete category with the arrow opposite to a frame homomorphism $h: L \rightarrow M$ represented by its right Galois adjoint $f=h_{*}: M \rightarrow L$ (uniquely determined by the fact that $h$ preserves all joins). Such f's will be referred to as localic maps.

1.1.2. By (dist), if $L$ is a frame and $a \in L$, the map $a \wedge(\cdot): L \rightarrow L$ preserves arbitrary joins and thus has a right adjoint $a \rightarrow(\cdot): L \rightarrow L$ determined by $c \leq a \rightarrow b$ iff $a \wedge c \leq b$. Hence, every frame is a Heyting algebra (note, however, that a frame homomorphism is not necessarily a Heyting one).

The pseudocomplement of $a \in L$ is $a^{*}=a \rightarrow 0$. We will use the standard facts like $a \leq b \Rightarrow b^{*} \leq a^{*}, a \leq a^{* *}$ or $a^{* * *}=a^{*}$.

1.1.3. Localic maps are just the infima preserving maps $f: L \rightarrow M$ (with left adjoint $f^{*}$ ) such that

$$
f[L \backslash\{1\}] \subseteq M \backslash\{1\} \text { and } f\left(f^{*}(a) \rightarrow b\right)=a \rightarrow f(b)
$$

for every $a \in M$ and $b \in L([22])$. 
1.1.4. We set $a \prec b$ for $a^{*} \vee b=1$. One speaks of a regular frame (or locale) if $a=\bigvee\{x \mid x \prec a\}$ for every $a$.

Further, let $\prec \prec$ be the largest interpolative relation (i.e. such that $a \prec \prec b \Rightarrow \exists c \in L: a \prec \prec c \prec \prec b)$ contained in $\prec$. One speaks of a completely regular frame (or locale) if $a=\bigvee\{x \mid x \prec \prec a\}$ for every $a$.

1.2. Down-set frame. The down-set frame $\mathfrak{D} L$ of a frame $L$ is the frame $\{U \subseteq L \mid \emptyset \neq U=\downarrow U\}$ (where $\downarrow U=\{x \mid x \leq a \in U\}$, as usual) with meets and joins given respectively by intersections and unions. The mapping

$$
\lambda^{\prime}=(a \mapsto \downarrow a): L \rightarrow \mathfrak{D} L
$$

is a localic map (it is the right adjoint to $v=(U \mapsto \bigvee U): \mathfrak{D} L \rightarrow L)$. Note that for any meet homomorphism $\phi: L \rightarrow M$ there is a unique frame homomorphism $g: \mathfrak{D} L \rightarrow M$ such that $g \lambda^{\prime}=\phi$, given by $g(U)=$ $\bigvee\{\phi(u) \mid u \in U\}$.

1.3. Taking quotients of a frame (saturation). For a relation $R \subseteq$ $L \times L$ on a frame $L$, call an $s \in L$ saturated (more precisely, $R$-saturated) if

$$
a R b \Rightarrow \forall c, a \wedge c \leq s \text { iff } b \wedge c \leq s .
$$

As any meet of saturated elements is saturated, we have a monotone mapping $\kappa=(x \mapsto \kappa(x))=\bigwedge\{s \mid x \leq s, s$ saturated $\}$ satisfying $x \leq \kappa(x), \kappa \kappa(x)=\kappa(x)$ and, moreover, $\kappa(x \wedge y)=\kappa(x) \wedge \kappa(y)$ (the nucleus of $R$ ), and if we set

$$
L / R=\{x \mid x=\kappa(x)\}
$$

we obtain a frame homomorphism $\kappa^{\prime}=(x \mapsto \kappa(x)): L \rightarrow L / R$ such that

(1.3.1) $x R y \Rightarrow \kappa^{\prime}(x)=\kappa^{\prime}(y)$, and

(1.3.2) if for a frame homomorphism $h: L \rightarrow K$ one has $x R y \Rightarrow$ $h(x)=h(y)$ then there is precisely one frame homomorphism $\bar{h}: L / R \rightarrow K$ such that $\bar{h} \cdot \kappa^{\prime}=h$; moreover, for $x \in L / R$, $\bar{h}(x)=h(x)$

(see e.g. [22]).

1.4. Sublocales. Subentities of locales (viewed as "generalized subspaces") are represented by sublocales. A subset $S$ of a frame $L$ is a sublocale of $L$ if, for any $A \subseteq S, x \in L$ and $a \in S$, we have $\bigwedge A \in S$ (in particular, $1 \in S$ ) and $x \rightarrow a \in S$. Sublocales are precisely such subsets for which the embedding map $j: S \rightarrow L$ is a (one-to-one) localic map (recall 1.1.1 and 1.1.3) — in fact, extremal monomorphisms in Loc. 
The set $\mathcal{S}(L)$ of all sublocales of $L$ forms a coframe (i.e., a complete lattice satisfying the dual of (dist)) under inclusion, in which arbitrary infima coincide with intersections, $\{1\}$ is the bottom element and $L$ is the top element. When $S$ is a sublocale of $L$ we also say that $L$ is a superlocale of $S$.

For more about frames and locales see e.g. [15, 22].

\section{Technique of entourages}

2.1. Coproduct ideals. For any frame $L$, take the Cartesian product $L \times L$ as a poset and consider the frame $\mathfrak{D}(L \times L)$. Call $U \in \mathfrak{D}(L \times L)$ a coproduct ideal (shortly, a $c p$-ideal) if

(2.1.1) for any $A \subseteq L$ and $b \in L$, if $A \times\{b\} \subseteq U$ then $(\bigvee A, b) \in U$, and

(2.1.2) for any $a \in L$ and $B \subseteq L$, if $\{a\} \times B \subseteq U$ then $(a, \bigvee B) \in U$.

Note that cp-ideals are precisely the elements in $\mathfrak{D}(L \times L)$ saturated with respect to the relation

$$
R=\left\{\left(\bigcup_{x \in A} \downarrow(x, b), \downarrow(\bigvee A, b)\right),\left(\bigcup_{y \in B} \downarrow(a, y), \downarrow(a, \bigvee B)\right) \mid A, B \subseteq L, a, b \in L\right\}
$$

The sets $A$ and $B$ in (2.1.1)-(2.1.2) can be void; hence, in particular, each cp-ideal contains as a subset $\mathrm{n}=\{(0, b),(a, 0) \mid a, b \in L\}$. It is easy to check that for each $(a, b) \in L \times L$,

$$
a \oplus b=\downarrow(a, b) \cup \mathrm{n}
$$

is a cp-ideal.

Intersections of cp-ideals are obviously cp-ideals and thus we have a complete lattice, which is indeed a frame; however, unions of cpideals are not necessarily cp-ideals and their suprema in this frame are typically bigger.

2.2. Binary products. Let $L$ be a frame. Recall (see e.g. [15, 22]; it should be noted that the first construction of frame coproducts appeared in [9]) that the coproduct $L \oplus L$ in Frm (product in Loc) is just the frame

$$
L \oplus L=\{U \in \mathfrak{D}(L \times L) \mid U \text { is a cp-ideal }\}
$$

with coproduct injections

$$
\iota_{1}=(a \mapsto a \oplus 1): L \rightarrow L \oplus L, \quad \iota_{2}=(b \mapsto 1 \oplus b): L \rightarrow L \oplus L .
$$

Note that for each $U \in L \oplus L$,

$$
U=\bigvee\{a \oplus b \mid(a, b) \in U\}=\bigcup\{a \oplus b \mid(a, b) \in U\}
$$


Notation. Using the symbol $L \times L$ when speaking of $L \oplus L$ as a product in the category Loc would probably obscure the matter. Therefore, we will keep the notation $L \oplus L$ also in Loc. We only have to keep in mind that then the injections $\iota_{1}, \iota_{2}$ become projections $p_{1}, p_{2}: L \oplus L \rightarrow L$.

2.3. Composition of decreasing binary relations. For any $U \in$ $\mathfrak{D}(L \times L)$ let

$$
\kappa_{0}(U)=\{(\bigvee A, b),(a, \bigvee B) \mid A \times\{b\} \subseteq U,\{a\} \times B \subseteq U\}
$$

and let $\langle U\rangle=\bigvee\{a \oplus b \mid(a, b) \in U\}$ be the cp-ideal generated by $U$. Of course, $U$ is a cp-ideal iff $\langle U\rangle=U$ iff $\kappa_{0}(U)=U$.

For any $U, V \in \mathfrak{D}(L \times L)$, the composite $U \circ V$ is the cp-ideal

$$
U \circ V=\bigvee\{a \oplus b \mid \exists c \neq 0, \quad(a, c) \in U \text { and }(c, b) \in V\},
$$

that is, the cp-ideal generated by the down-set

$$
U \cdot V=\{(a, b) \mid \exists c \neq 0, \quad(a, c) \in U \text { and }(c, b) \in V\} .
$$

It is easy to check that

$$
\kappa_{0}(U) \cdot V \subseteq \kappa_{0}(U \cdot V)
$$

(and, by symmetry, we also have $U \cdot \kappa_{0}(V) \subseteq \kappa_{0}(U \cdot V)$ ).

The following technical lemma plays an important role in the entourage approach to frame uniformities (cf. [19, Lemma 3.1]).

2.3.1. Lemma. For any $U, V \in \mathfrak{D}(L \times L),\langle U\rangle \circ\langle V\rangle=U \circ V$.

Proof. It suffices to show that $\langle U\rangle \cdot\langle V\rangle \subseteq\langle U \cdot V\rangle$. For that, consider the non-empty set

$$
\mathbb{D}=\{S \in \mathfrak{D}(L \times L) \mid U \subseteq S \subseteq\langle U\rangle, S \cdot V \subseteq\langle U \cdot V\rangle\} .
$$

By $(*), S \in \mathbb{D}$ implies $\kappa_{0}(S) \in \mathbb{D}$. On the other hand, for any non-void subset of $\mathbb{D}$, its union is also in $\mathbb{D}$ and thus $\mathbb{D}$ has a largest element $D$. But $\kappa_{0}(D) \in \mathbb{D}$ so $D=\kappa_{0}(D)$, i.e., $D$ is a cp-ideal. Hence $\langle U\rangle=D \in \mathbb{D}$, that is, $\langle U\rangle \cdot V \subseteq\langle U \cdot V\rangle$, and by symmetry, $U \cdot\langle V\rangle \subseteq\langle U \cdot V\rangle$. Then the inclusion $\langle U\rangle \cdot\langle V\rangle \subseteq\langle U \cdot V\rangle$ follows since $\langle\cdot\rangle$ is an idempotent operator.

2.4. Entourages. An entourage in a frame $L$ is an element $E \in L \oplus L$ such that

$$
\bigvee\{u \mid(u, u) \in E\}=1 .
$$

2.4.1. Lemma. If $E$ is an entourage then $E \leq E \circ E$. 
Proof. Let $(a, b) \in E$ with $b \neq 0$. We have

$$
b=\bigvee\{b \wedge u \mid(u, u) \in E\}=\bigvee\{b \wedge u \mid(u, u) \in E, u \wedge b \neq 0\} .
$$

Now we have, for $b \wedge u \neq 0, a \oplus(b \wedge u) \leq E$ and $(b \wedge u) \oplus(b \wedge u) \leq E$.

Hence $a \oplus(b \wedge u) \leq E \circ E$ and, finally,

$a \oplus b=a \oplus \bigvee\{b \wedge u \mid(u, u) \in E\}=\bigvee\{a \oplus(b \wedge u) \mid(u, u) \in E\} \leq E \circ E$

2.5. For each $x \in L$ and $E \in L \oplus L$, let

$$
\begin{aligned}
& E_{1} x=\bigvee\{a \in L \mid(a, b) \in E, b \wedge x \neq 0\} \\
& E_{2} x=\bigvee\{b \in L \mid(a, b) \in E, a \wedge x \neq 0\}
\end{aligned}
$$

and

$$
E x=\bigvee\{a \in L \mid(a, a) \in E, a \wedge x \neq 0\} .
$$

The following formulas are easy to check (for details see [19, 21]):

(2.5.1) $F_{1}\left(E_{1} x\right) \leq(F \circ E)_{1} x$ and $F_{2}\left(E_{2} x\right) \leq(E \circ F)_{2} x$.

(2.5.2) If $E$ is an entourage then $x \leq E x \leq E_{1} x \wedge E_{2} x$.

$(2.5 .3)\langle E\rangle_{i} x=E_{i} x(i=1,2)$.

2.5.1. Lemma. For each entourage $E$, we have:

(a) $E x^{* *}=E x \quad$ and $\quad E_{i} x^{* *}=E_{i} x \quad(i=1,2)$.

(b) $(E x, E x) \in E^{3}$ for any $(x, x) \in E$.

(c) $\left(E_{1} x, E_{2} y\right) \in E^{3}$ for any $(x, y) \in E$.

Proof. (a) Follows immediately from the fact that $y \wedge x=0$ iff $y \wedge x^{* *}=$ 0 .

(b) If $(y, y),(x, x),(z, z) \in E$ with $y \wedge x \neq 0$ and $x \wedge z \neq 0$, then $(y, z) \in E^{3}$.

(c) If $(a, b),(x, y),(c, d) \in E$ with $b \wedge x \neq 0$ and $y \wedge c \neq 0$, then $(a, d) \in E^{3}$.

2.6. For an entourage $E$ set

$$
\widetilde{E}=\bigvee\left\{x^{* *} \oplus y^{* *} \mid(x, y) \in E\right\} .
$$

Fact. This is clearly an entourage of $L$ and $\widetilde{E} \leq E^{3}$.

Indeed, by (a) and (c) above we have, for any $(x, y) \in E$,

$$
\left(x^{* *}, y^{* *}\right) \leq\left(E_{1} x^{* *}, E_{2} y^{* *}\right)=\left(E_{1} x, E_{2} y\right) \in E^{3} .
$$




\section{UNIFORMITIES AND QUASI-UNIFORMITIES}

3.1. Entourage uniformities. For an entourage $E$ (resp. a set of entourages $\mathcal{E}$ ) write

$$
b \triangleleft_{E} a \text { if } E \circ(b \oplus b) \leq a \oplus a, \quad \text { and } \quad b \triangleleft_{\mathcal{E}} a \text { if } \exists E \in \mathcal{E}, b \triangleleft_{E} a .
$$

A system of entourages $\mathcal{E}$ is said to be admissible if

$$
\forall a \in L, \quad a=\bigvee\left\{b \mid b \triangleleft_{\mathcal{E}} a\right\} .
$$

Further, for an entourage $E$ set

$$
E^{-1}=\{(a, b) \mid(b, a) \in E\}
$$

(which is obviously an entourage again).

3.1.1. Definition. A (entourage) uniformity [19] on a frame $L$ is an admissible system of entourages $\mathcal{E}$ such that

(E1) $E \in \mathcal{E}$ and $E \leq F \Rightarrow F \in \mathcal{E}$,

(E2) $E, F \in \mathcal{E} \Rightarrow E \cap F \in \mathcal{E}$,

(E3) for every $E \in \mathcal{E}$ there is an $F \in \mathcal{E}$ such that $F \circ F \leq E$, and

(E4) $E \in \mathcal{E} \Rightarrow E^{-1} \in \mathcal{E}$.

A uniform frame (or locale) is a pair $(L, \mathcal{E})$ where $\mathcal{E}$ is a uniformity on $L$. In particular, the admissibility condition (3.1.1) implies that $L$ is always regular (in fact, completely regular).

A basis $\mathcal{B}$ of a uniformity is an admissible system of entourages satisfying (E3), (E4) and

(E2') $E, F \in \mathcal{B} \Rightarrow \exists G \in \mathcal{B}, G \leq E \cap F$.

A uniformity is then generated by taking all the $F \geq E$ with $E \in \mathcal{B}$.

3.2. Non-symmetric uniformities. For a system $\mathcal{E}$ of entourages define, first, $\overline{\mathcal{E}}$ as the filter of entourages generated by $\mathcal{E} \cup\left\{E^{-1} \mid E \in \mathcal{E}\right\}$ (of course, if $\mathcal{E}$ is a uniformity, $\overline{\mathcal{E}}=\mathcal{E}$ ). If $\mathcal{E}$ satisfies (E1), (E2) and (E3) and $\overline{\mathcal{E}}$ is admissible we speak of $\mathcal{E}$ as of a quasi-uniformity and of $(L, \mathcal{E})$ as of a quasi-uniform frame $([20,21])$. A basis $\mathcal{B}$ of a quasi-uniformity is a system satisfying (E2') and (E3) such that $\overline{\mathcal{B}}$ is admissible.

Because of the lack of symmetry condition (E4), the system $\mathcal{E}$ unmodified induces two distinct order relations:

$$
\begin{aligned}
& x \triangleleft_{\mathcal{E}}^{1} y \equiv \exists E \in \mathcal{E}, E \circ(x \oplus x) \leq y \oplus y, \\
& x \triangleleft_{\mathcal{E}}^{2} y \equiv \exists E \in \mathcal{E}, \quad(x \oplus x) \circ E \leq y \oplus y .
\end{aligned}
$$

Note that $x \triangleleft_{\mathcal{E}}^{i} y$ iff $E_{i} x \leq y$ for some $E \in \mathcal{E}(i=1,2)$. 
3.2.1. Notes. (1) Let $\mathcal{E}$ be a quasi-uniformity on a frame $L$. Then $\mathcal{E}^{-1}=\left\{E^{-1} \mid E \in \mathcal{E}\right\}$ is also a quasi-uniformity (the conjugate quasiuniformity on $L$ ) and $\overline{\mathcal{E}}$ is the coarsest uniformity containing $\mathcal{E}$.

(2) The two relations $\triangleleft_{\mathcal{E}}^{1}$ and $\triangleleft_{\mathcal{E}}^{2}$ yield two subframes of $L$, namely

$$
L_{i}(\mathcal{E})=\left\{x \in L \mid x=\bigvee\left\{y \in L \mid y \triangleleft_{\mathcal{E}}^{i} x\right\}\right\} \quad(i=1,2) .
$$

(3) Recall that a biframe is a triple $\left(L, L_{1}, L_{2}\right)$ of frames where $L_{1}$ and $L_{2}$ are subframes of $L$ and

$$
\forall a \in L, a=\bigvee\left\{a_{1} \wedge a_{2} \mid a_{i} \in L_{i}, a_{1} \wedge a_{2} \leq a\right\} .
$$

Biframes are the pointfree counterpart of bitopological spaces ([5]).

The admissibility condition on a quasi-uniform frame $(L, \mathcal{E})$ means precisely that the triple $\left(L, L_{1}(\mathcal{E}), L_{2}(\mathcal{E})\right)$ is a (completely regular) biframe (see [20, Remark 3.4]). Thus every quasi-uniformity on a frame $L$ induces a natural biframe structure on $L$, in the same way as every quasi-uniformity on a set $X$ endows $X$ with a bitopological structure $([10])$.

3.3. Proposition. Let $\mathcal{E}$ be a quasi-uniformity on a frame L. If $b_{1} \triangleleft_{\mathcal{E}}^{1} a_{1}$ and $b_{2} \triangleleft_{\mathcal{E}}^{2} a_{2}$ then $\left(b_{1} \wedge b_{2}\right) \triangleleft \overline{\mathcal{E}}\left(a_{1} \wedge a_{2}\right)$.

Proof. If $E \circ\left(b_{1} \oplus b_{1}\right) \leq a_{1} \oplus a_{1}$ and $\left(b_{2} \oplus b_{2}\right) \circ F \leq a_{2} \oplus a_{2}$ then $F^{-1} \circ\left(b_{2} \oplus b_{2}\right) \leq a_{2} \oplus a_{2}$. Hence $(E \cap F)^{-1} \circ\left(b_{1} \wedge b_{2} \oplus b_{1} \wedge b_{2}\right) \leq$ $a_{1} \wedge a_{2} \oplus a_{1} \wedge a_{2}$.

3.4. Let $\mathcal{E}$ be a quasi-uniformity on $L$. For $E \in \mathcal{E}$ set

$$
E^{\triangleleft}=\bigvee\left\{x \oplus y \mid x \triangleleft_{\mathcal{E}}^{1} a, y \triangleleft_{\mathcal{E}}^{2} b,(a, b) \in E\right\} \leq E .
$$

3.4.1. Proposition. For any $F$ such that $F^{3} \leq E, F \leq E^{\triangleleft}$. Hence $E^{\triangleleft} \in \mathcal{E}$ and $\left\{E^{\triangleleft} \mid E \in \mathcal{E}\right\}$ is a basis for $\mathcal{E}$.

Proof. Let $(x, y) \in F$. By $2.5(\mathrm{c}),\left(F_{1} x, F_{2} y\right) \in F^{3} \leq E$, and, of course, $x \triangleleft_{\mathcal{E}}^{1} F_{1} x$ as well as $y \triangleleft_{\mathcal{E}}^{2} F_{2} y$.

3.5. The fact in 2.6 provides an alternative basis for $\mathcal{E}$ :

3.5.1. Proposition. Let $\mathcal{E}$ be a quasi-uniformity on L. Then $\{\widetilde{E} \mid$ $E \in \mathcal{E}\}$ is a basis of $\mathcal{E}$.

3.6. Product maps. By the universal property of products, for each localic map $f: L \rightarrow M$ there is the (unique) localic map

$$
f \times f: L \oplus L \rightarrow M \oplus M
$$

such that $p_{i}(f \times f)=f p_{i}$ for $i=1,2$. Its left adjoint $(f \times f)^{*}$ in Frm is the unique frame homomorphism

$$
h \oplus h: M \oplus M \rightarrow L \oplus L
$$


such that $(h \oplus h) \iota_{i}=\iota_{i} h\left(\right.$ where $\left.h=f^{*}\right)$. Clearly,

$$
(h \oplus h)(E)=\bigvee_{(a, b) \in E}(h(a) \oplus h(b)) \text {. }
$$

Furthermore, for every $E \in L \oplus L$,

$$
(h \oplus h)\left(E^{-1}\right)=(h \oplus h)(E)^{-1} .
$$

Indeed, we have $(h \oplus h)\left(E^{-1}\right)=\bigvee\{h(a) \oplus h(b) \mid(b, a) \in E\}$ while

$$
(h \oplus h)(E)^{-1}=\bigvee\{x \oplus y \mid(y, x) \in(h \oplus h)(E)\}
$$

and, clearly, $(h \oplus h)\left(E^{-1}\right) \leq(h \oplus h)(E)^{-1}$. Regarding the reverse inclusion, it is equivalent to

$$
(h \oplus h)(E) \leq(h \oplus h)\left(E^{-1}\right)^{-1}
$$

and this follows immediately from the obvious

$$
\bigcup_{(a, b) \in E}(h(a) \oplus h(b)) \leq(h \oplus h)\left(E^{-1}\right)^{-1} .
$$

3.6.1. Uniform maps. If $(L, \mathcal{E})$ and $(M, \mathcal{F})$ are quasi-uniform locales and $f: L \rightarrow M$ is a localic map satisfying

$$
\forall F \in \mathcal{F}, \quad(f \times f)^{*}(F) \in \mathcal{E} .
$$

then we speak of a uniform map $f:(L, \mathcal{E}) \rightarrow(M, \mathcal{F})$ (and the corresponding left adjoint $h=f^{*}:(M, \mathcal{F}) \rightarrow(L, \mathcal{E})$ is called a uniform frame homomorphism). We then have the category QULoc of quasiuniform locales and uniform maps and its full subcategory ULoc of uniform locales.

3.6.2. Uniform embeddings. Let $j: L \rightarrow M$ be an embedding in Loc with adjoint onto frame homomorphism $h=j^{*}: M \rightarrow L$. Since regular monomorphisms are preserved by products, $j \times j$ is also oneto-one.

By a uniform embedding $j:(L, \mathcal{E}) \rightarrow(M, \mathcal{F})$ we mean a one-to-one uniform map such that

$$
\mathcal{E}=\left\{(j \times j)^{*}(F) \mid F \in \mathcal{F}\right\} .
$$

\section{Dense Uniform EMBEDDings}

4.1. Dense maps and dense sublocales. Recall that a frame homomorphism $h: L \rightarrow M$ is dense if

$$
h(x)=0 \Rightarrow x=0,
$$

and that a sublocale $S \subseteq L$ is dense if

$$
\bar{S}=L \text {, that is, } 0 \in S \text {. }
$$

Consider the localic map $f=h_{*}: M \rightarrow L$ associated with $h$. 
4.1.1. Proposition. The following are equivalent:

(i) $h$ is dense.

(ii) $f(0)=0$.

(iii) $f[M]$ is dense in $L$.

Proof. $(\mathrm{a}) \Leftrightarrow(\mathrm{b}): x \leq 0$ iff $h(x) \leq 0$ iff $x \leq f(0)$ makes $f(0)=0$. $(\mathrm{b}) \Leftrightarrow(\mathrm{c})$ is trivial.

4.1.2. Regarding uniform maps $f:(L, \mathcal{E}) \rightarrow(M, \mathcal{F})$ we will use the fact that $f$ is epic if it is dense, a consequence of the regularity of the frames involved $([22, \S \mathrm{V} .6 .5])$.

4.2. Proposition (Banaschewski [2]). Let $S \subseteq L$ be a dense sublocale of a regular locale. Then $S$ join-generates $L$.

In particular, if for $a, b \in L,\{x \mid x \in S, x \leq a\}=\{x \mid x \in S, x \leq b\}$, then $a=b$.

Proof. Since $x \prec a$ implies $x^{* *} \prec a\left(1=x^{*} \vee a=x^{* * *} \vee a\right)$, a regular frame is generated by its Booleanization $\mathfrak{B} L=\left\{x^{* *} \mid x \in L\right\}$, contained in every dense sublocale by Isbell's density theorem $([22, \S$ III.8.3].

4.3. From now on we will consider an embedding $j: L \rightarrow M$ with adjoint onto frame homomorphism $h=j^{*}: M \rightarrow L$. Since $j$ is oneto-one, we infer from $j h j=j$ that $h j=\mathrm{id}$. In case $j$ is dense (by the density we mean, of course, that $h$ is dense, that is, by 4.1.1, that $j(0)=0)$ then $j(x)=0$ iff $x=0$.

4.3.1. Proposition. For each $E \in L \oplus L$,

$$
(j \times j)(E)=\bigvee\{j(a) \oplus j(b) \mid(a, b) \in E\} .
$$

Proof. Let $E \in L \oplus L$. We have

$$
(j \times j)(E)=\bigvee\{F \in M \oplus M \mid(h \oplus h)(F) \leq E\} .
$$

The latter is equal to $\bigvee\{j(a) \oplus j(b) \mid(a, b) \in E\}$. Indeed:

$\geq$ :

$$
(h \oplus h)\left(\bigvee_{(a, b) \in E}(j(a) \oplus j(b))=\bigvee_{(a, b) \in E}(h j(a) \oplus h j(b))=\bigvee_{(a, b) \in E}(a \oplus b)=E\right.
$$

since $h j=\mathrm{id}$.

$\leq:(h \oplus h)(F) \leq E$ iff $\bigvee_{(x, y) \in F}(h(x) \oplus h(y)) \leq E$ and the latter clearly implies that $F \leq \bigvee_{(a, b) \in E}(j(a) \oplus j(b))$ since

$$
(x, y) \in F \Rightarrow(h(x), h(y)) \in E \Rightarrow(j h(x), j h(y)) \in \bigvee_{(a, b) \in E}(j(a) \oplus j(b))
$$

and $(x, y) \leq(j h(x), j h(y))$ 
Since $h j=\mathrm{id}$, we have immediately:

4.3.2. Corollary. For each $E \in L \oplus L,(h \oplus h)(j \times j)(E)=E$.

4.4. If $j$ is moreover dense, then $j h(x) \leq x^{* *}\left(\right.$ for $h\left(j h(x) \wedge x^{*}\right)=$ $h(x) \wedge h\left(x^{*}\right)=h(0)=0$ implies $\left.j h(x) \wedge x^{*}=0\right)$, and therefore

$$
\bigcup_{(a, b) \in E}(j h(a) \oplus j h(b)) \leq \widetilde{E} .
$$

Hence:

4.4.1. Corollary. Let $j: L \rightarrow M$ be a dense embedding. For each $F \in M \oplus M, \quad F \leq(j \times j)(h \oplus h)(F) \leq \widetilde{F}$.

4.5. Proposition. Let $j:(L, \mathcal{E}) \rightarrow(M, \mathcal{F})$ be a uniform map with left adjoint $h=j^{*}$. Then $j$ is a dense uniform embedding if and only if

$$
\{(j \times j)(E) \mid E \in \mathcal{E}\} \text { is a basis of } \mathcal{F}
$$

and

$$
(h \oplus h)(j \times j)(E)=E \text { for every } E \in L \oplus L .
$$

Proof. $\Rightarrow$ : By definition of a uniform embedding,

$$
\mathcal{E}=\left\{(j \times j)^{*}(F)=(h \oplus h)(F) \mid F \in \mathcal{F}\right\}
$$

and hence $(j \times j)(E)=(j \times j)(h \oplus h)(F) \geq F$ for some $F \in \mathcal{F}$, which shows that every $(j \times j)(E)$ is an entourage in $\mathcal{F}$. Now for each $F \in \mathcal{F}$ take $G \in \mathcal{F}$ such that $\widetilde{G} \leq F$ (recall Proposition 2.2). By 4.4.1,

$$
G \leq(j \times j)(h \oplus h)(G) \leq \widetilde{G} \leq F .
$$

This proves condition (1) while (2) was already proved in 4.3.2.

$\Leftarrow$ : We need to show that $\mathcal{E}=\{(h \oplus h)(F) \mid F \in \mathcal{F}\}$. If $E \in \mathcal{E}$ then $E=(h \oplus h)(j \times j)(E)$ by $(2)$, and $(j \times j)(E) \in \mathcal{F}$ by $(1)$. Conversely, condition (1) ensures that each $(h \oplus h)(F)$ contains some $(h \oplus h)(j \times j)(E)=E \in \mathcal{E}$.

4.6. Proposition. Let $j:(L, \mathcal{E}) \rightarrow(M, \mathcal{F})$ be a dense uniform embedding with left adjoint $h: M \rightarrow L$. Then, for every $x \in L$ and $y \in M$ :

(1) $h(y) \triangleleft_{\mathcal{E}}^{i} x$ iff $y \triangleleft_{\mathcal{F}}^{i} j(x)(i=1,2)$.

(2) $h(y) \triangleleft_{\overline{\mathcal{E}}} x$ iff $y \triangleleft_{\overline{\mathcal{F}}} j(x)$.

Proof. (1) $\Rightarrow$ : If $h(y) \triangleleft_{\mathcal{E}}^{1} x$ then there is some $E \in \mathcal{E}$ such that $E_{1} h(y) \leq$ $x$ (recall 2.5). Take $F=(j \times j)(E) \in \mathcal{F}$ (by the previous proposition). By (2.5.3),

$$
F_{1} y=\bigvee\left\{a \mid(a, b) \in \bigcup_{\left(a^{\prime}, b^{\prime}\right) \in E}\left(j\left(a^{\prime}\right) \oplus j\left(b^{\prime}\right)\right), b \wedge y \neq 0\right\}
$$


and therefore for each such $(a, b)$ we have $(a, b) \leq\left(j\left(a^{\prime}\right), j\left(b^{\prime}\right)\right)$ for some $\left(a^{\prime}, b^{\prime}\right) \in E$. Then $0 \neq b \wedge y \leq j\left(b^{\prime}\right) \wedge j h(y)=j\left(b^{\prime} \wedge h(y)\right)$ implies $b^{\prime} \wedge h(y) \neq 0$ and thus $a^{\prime} \leq E_{1} h(y) \leq x$ and hence $a \leq j\left(a^{\prime}\right) \leq j(x)$. In conclusion, $F_{1} y \leq j(x)$.

$\Leftarrow$ : If $y \triangleleft_{\mathcal{F}}^{1} j(x)$ apply the preceding proposition to get some $E \in \mathcal{E}$ such that

$$
((j \times j)(E))_{i} y \leq j(x) .
$$

Then, if $(a, b) \in E$ with $b \wedge h(y) \neq 0$, that is, $h j(b) \wedge h(y) \neq 0$, we have $(j(a), j(b)) \in(j \times j)(E)$ and $j(b) \wedge y \neq 0$. Hence $j(a) \leq j(x)$, and since $j$ is an embedding, $a \leq x$. Hence $h(y) \triangleleft_{\mathcal{E}}^{1} x$.

(2) can be proved similarly.

4.7. Let $j: L \rightarrow M$ be a dense embedding. Using the formula in 4.3.1, a proof similar to the one for frame homomorphisms in (3.6.2) gives us the analogous property for $j \times j$ :

$$
\text { for every } E \in L \oplus L,(j \times j)\left(E^{-1}\right)=(j \times j)(E)^{-1} \text {. }
$$

It follows that for any dense uniform embedding $j:(L, \mathcal{E}) \rightarrow(M, \mathcal{F})$,

$$
(j \times j)\left(E_{1} \cap E_{2}^{-1}\right)=(j \times j)\left(E_{1}\right) \cap(j \times j)\left(E_{2}\right)^{-1}
$$

and thus that

$$
(j \times j)(E) \in \overline{\mathcal{F}} \text { for every } E \in \overline{\mathcal{E}} \text {. }
$$

Furthermore,

$$
F \circ F \leq E \Rightarrow(j \times j)(F) \circ(j \times j)(F) \leq(j \times j)(E) .
$$

Indeed, by $2.3,(j \times j)(F) \circ(j \times j)(F)$ is equal to

$$
\bigcup\{j(a) \oplus j(b) \mid(a, b) \in F\} \circ \bigcup\{j(a) \oplus j(b) \mid(a, b) \in F\} .
$$

If $(a, b),(c, d) \in F$ with $j(b) \wedge j(c) \neq 0$, then $b \wedge c \neq 0$ (since $j(0)=0$ by density) and therefore $(a, d) \in F \circ F \leq E$. Hence $(j(a), j(d)) \in$ $(j \times j)(E)$.

4.7.1. Proposition. Let $(L, \mathcal{E})$ and $(M, \mathcal{F})$ be quasi-uniform frames. If $j:(L, \mathcal{E}) \rightarrow(M, \mathcal{F})$ is a dense uniform embedding, then $j:(L, \overline{\mathcal{E}}) \rightarrow$ $(M, \overline{\mathcal{F}})$ is a dense uniform embedding.

Proof. We need to show that for $h=j^{*}$,

$$
\{(h \oplus h)(F) \mid F \in \overline{\mathcal{F}}\}=\overline{\mathcal{E}} .
$$

$\subseteq$ : For each $F \in \overline{\mathcal{F}}$ let $G_{1}, G_{2} \in \mathcal{F}$ such that $G_{1} \cap G_{2}^{-1} \subseteq F$. Then, using (3.6.2), we get

$$
(h \oplus h)(F) \supseteq(h \oplus h)\left(G_{1} \cap G_{2}^{-1}\right)=(h \oplus h)\left(G_{1}\right) \cap(h \oplus h)\left(G_{2}\right)^{-1} \in \overline{\mathcal{E}} .
$$


$\supseteq$ : Let $E \in \overline{\mathcal{E}}$. By 4.3.2, $E=(h \oplus h)(j \times j)(E)$, where $(j \times j)(E) \in \overline{\mathcal{F}}$ by $(4.7 .2)$.

4.7.2. Proposition. Let $(L, \mathcal{E})$ be a quasi-uniform frame. If

$$
j:(L, \overline{\mathcal{E}}) \rightarrow(M, \mathcal{F})
$$

is a dense uniform embedding between uniform frames, then

$$
\mathcal{B}=\{(j \times j)(E) \mid E \in \mathcal{E}\}
$$

is a basis for a quasi-uniformity in $M$.

Proof. Let $E, F \in \mathcal{E}$. By (4.7.1),

$$
(j \times j)(E) \cap(j \times j)(F)=(j \times j)(E \cap F) \in \mathcal{B} .
$$

Furthermore, for $(j \times j)(E) \in \mathcal{B}$, consider $F \in \mathcal{E}$ such that $F \circ F \leq E$. By (4.7.3),

$$
(j \times j)(F) \circ(j \times j)(F) \leq(j \times j)(E) .
$$

Finally, regarding admissibility, we need to show that

$$
b=\bigvee\left\{y \in M \mid y \triangleleft_{\overline{\mathcal{B}}} b\right\}
$$

for every $b \in M$. Of course, by the admissibility of $\mathcal{F}$ in $M$, it suffices to check that $y \triangleleft_{\mathcal{F}} b$ implies $y \triangleleft_{\overline{\mathcal{B}}} b$. Let $F \in \mathcal{F}$ such that $F \circ(y \oplus y) \leq$ $b \oplus b$ and apply 3.5.1 to get some $G \in \mathcal{F}$ such that $\widetilde{G} \leq F$. Then $(h \oplus h)(G) \in \overline{\mathcal{E}}$, that is, $(h \oplus h)(G) \geq E \cap E^{-1}$ for some $E \in \mathcal{E}$. By (4.7.1),

$$
(j \times j)\left(E \cap E^{-1}\right)=(j \times j)(E) \cap(j \times j)(E)^{-1} \in \overline{\mathcal{B}} .
$$

Moreover, by 4.4.1,

$$
\begin{aligned}
(j \times j)\left(E \cap E^{-1}\right) \circ(y \oplus y) & \leq(j \times j)(h \oplus h)(G) \circ(y \oplus y) \\
& \leq \widetilde{G} \circ(y \oplus y) \leq F \circ(y \oplus y) \leq b \oplus b .
\end{aligned}
$$

\section{Cauchy maps, and a Universal one}

5.1. Cauchy and weak Cauchy maps. Let $(L, \mathcal{E})$ be a quasi-uniform frame (or, more particularly, a uniform frame) and let $M$ be a frame. A Cauchy map $\phi:(L, \mathcal{E}) \rightarrow M$ is a bounded meet homomorphism $\phi: L \rightarrow M$ such that

(C1) for all $E \in \mathcal{E}, \bigvee\{\phi(a) \mid(a, a) \in E\}=1$, and

(C2) for each $a \in L, \phi(a)=\bigvee\left\{\phi(b) \mid b \triangleleft_{\overline{\mathcal{E}}} a\right\}$.

If we do not require (C2) we speak of a weak Cauchy map. 
5.1.1. Notes. (1) Recall the Cauchy points from classical completions of uniform spaces $(X, \mathcal{E})$, that is, filters $F$ in $L=\Omega(X)$ such that $F \cap\{a \mid(a, a) \in E\} \neq \emptyset^{1}$, for all $E \in \mathcal{E}$, and for every $a \in F$ there is a $b \in F$ such that $b \triangleleft_{\mathcal{E}} a$.

Taking their characteristic maps we have Cauchy points represented as bounded meet semilattice homomorphisms $\phi:(L, \mathcal{E}) \rightarrow \mathbf{2}=\{0,1\}$ such that

- for all $E \in \mathcal{E}, \bigvee\{\phi(a) \mid(a, a) \in E\}=1$, and

- for every $a \in L, \phi(a)=\bigvee\left\{\phi(b) \mid b \triangleleft_{\mathcal{E}} a\right\}$.

Thus, Cauchy maps are Cauchy points with a general frame $M$ instead of 2.

(2) In the standard terminology one speaks of regular Cauchy maps, and the term "Cauchy map" is preserved for what we call "weak Cauchy map". We have decided to simplify the terminology using the shorter expression for the fundamental concept, and the longer one for the technical modification.

5.2. Observations. (1) If $\phi$ is Cauchy and $h$ a frame homomorphism then $h \phi$ is Cauchy.

(2) If $\phi$ is weakly Cauchy and $h$ a uniform frame homomorphism then $\phi h$ is weakly Cauchy.

5.2.1. Note. The statement (2) does not hold for Cauchy maps. An example will be given in 6.5.2.

5.3. $\mathbf{C}(L, \mathcal{E})$ and the mapping $\lambda:(L, \mathcal{E}) \rightarrow \mathbf{C}(L, \mathcal{E})$. Recall the mapping $\lambda^{\prime}=(a \mapsto \downarrow a): L \rightarrow \mathfrak{D}(L)$ and the fact that a meet homomorphism $\phi: L \rightarrow M$ is uniquely extendable to a frame homomorphism $g: \mathfrak{D}(L) \rightarrow M$ such that $g \lambda^{\prime}=\phi$, namely the one given by $g(U)=\bigvee\{\phi(u) \mid u \in U\}$.

Defining on $\mathfrak{D}(L)$ the relation

$$
R_{\mathcal{E}}=\left\{(\bigcup\{\downarrow a \mid(a, a) \in E\}, L),\left(\bigcup\left\{\downarrow x \mid x \triangleleft_{\overline{\mathcal{E}}} y\right\}, \downarrow y\right) \mid E \in \mathcal{E}, y \in L\right\}
$$

we obtain a frame

$$
\mathbf{C}(L, \mathcal{E})=\mathfrak{D}(L) / R_{\mathcal{E}}
$$

It is easy to check that the saturated sets constituting this frame (they will be referred to as Cauchy ideals in $(L, \mathcal{E}))$ are the $U \in \mathfrak{D}(L)$ such that

(R1) if for some $E \in \mathcal{E}, E \wedge\{x\}=\{a \wedge x \mid(a, a) \in E\} \subseteq U$, then $x \in U$, and

\footnotetext{
${ }^{1}$ Recall 2.4; the sets $\{a \in L \mid(a, a) \in E\}$ are the covers replacing entourages in the covering description of a uniformity, see e.g. [21].
} 
(R2) if $\left\{x \mid x \triangleleft_{\overline{\mathcal{E}}} y\right\} \subseteq U$ then $y \in U$.

The composite of $\lambda^{\prime}$ with the frame homomorphism $\mathfrak{D}(L) \rightarrow \mathbf{C}(L, \mathcal{E})$ will be denoted by $\lambda_{(L, \mathcal{E})}$.

5.3.1. Note. $\mathbf{C}(L, \mathcal{E})$ was used in several texts (e.g., $[3,2,4,22])$ (typically for the cover uniformities) as an underlying frame of the completion of $(L, \mathcal{E})$.

In fact it is a special case of a general construction of a frame extension of a site (see [1]). A site is a meet semilattice (here a frame) together with a covering, a subset $\sqsubseteq$ of $L \times \mathfrak{D}(L) ;(x, U) \in \sqsubseteq$ means that we want to force $x$ to become a supremum of $U$. In our case the covering is constituted by the couples

$$
(L,\{\downarrow a \mid(a, a) \in E\}), E \in \mathcal{E}, \quad \text { and } \quad(\downarrow y,\{\downarrow x \mid x \triangleleft \overline{\mathcal{E}} y\}), y \in L .
$$

This site covering makes in a canonical way $\lambda:(L, \mathcal{E}) \rightarrow \mathbf{C}(L, \mathcal{E})$ a Cauchy map, and the following very easy theorem comes as no surprise. In the next section we will then show that a completion procedure, symmetric or not, can be obtained as an easy consequence.

5.4. Theorem. $\lambda_{(L, \mathcal{E})}$ is a Cauchy map and it is a universal Cauchy map in the sense that for every Cauchy map $\phi:(L, \mathcal{E}) \rightarrow M$ there is precisely one frame homomorphism $h: \mathbf{C}(L, \mathcal{E}) \rightarrow M$ such that the diagram

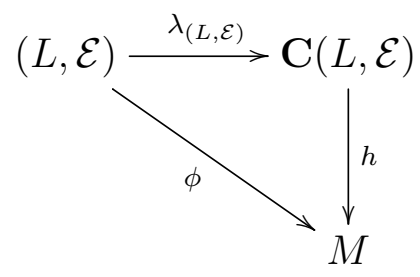

commutes.

Proof. $\lambda_{(L, \mathcal{E})}$ is obviously Cauchy by the definition of the relation $R_{\mathcal{E}}$ above.

Now let $\phi:(L, \mathcal{E}) \rightarrow M$ be Cauchy. It is a bounded meet homomorphism and hence we have the frame homomorphism $g: \mathfrak{D}(L) \rightarrow M$ as in 5.3. We have

$$
\begin{aligned}
& g(\bigcup\{\downarrow a \mid(a, a) \in E\})=\bigvee\{\phi(a) \mid(a, a) \in E\}=1, \text { for } E \in \mathcal{E}, \quad \text { and } \\
& g\left(\bigcup\left\{\downarrow x \mid x \triangleleft_{\overline{\mathcal{E}}} y\right\}\right)=\bigvee\left\{\phi(x) \mid x \triangleleft_{\overline{\mathcal{E}}} y\right\}=\phi(y)=g(\downarrow y),
\end{aligned}
$$

hence $g$ respects the relation $R_{\mathcal{E}}$ and restricts to a frame homomorphism $h: \mathbf{C}(L, \mathcal{E}) \rightarrow M$.

5.5. Proposition. Each dense uniform embedding $j:(L, \mathcal{E}) \subseteq(M, \mathcal{F})$ is a Cauchy map. 
Proof. (C1) is obvious. For (C2) take an arbitrary $a$ in $L$. We need to show that $j(a)=\bigvee\left\{j(x) \mid x \triangleleft_{\overline{\mathcal{E}}} a\right\}$. By the admissibility of $\overline{\mathcal{F}}$, $j(a)=\bigvee\{b \in M \mid b \triangleleft \overline{\mathcal{F}} j(a)\}$. Let $h=j^{*}: M \rightarrow L$. Then, by 4.6, $j(a)=\bigvee\left\{b \in M \mid h(b) \triangleleft_{\overline{\mathcal{E}}} a\right\}$, and this supremum is clearly equal to $\bigvee\{j(x) \mid x \triangleleft \overline{\mathcal{E}} a\}$ since $b \leq j h(b)$, for every $b \in M$, and $h j(x)=x$, for every $x \in L$.

\section{Applications: Completion}

6.1. Completions. A (quasi-)uniform locale $(L, \mathcal{E})$ is said to be complete if every dense uniform embedding with domain $(L, \mathcal{E})$ is an isomorphism.

Let $(L, \mathcal{E})$ and $(M, \mathcal{F})$ be (quasi-)uniform locales with $(M, \mathcal{F})$ complete. Then $(M, \mathcal{F})$ is a completion of $(L, \mathcal{E})$ provided that there is a dense uniform embedding

$$
j:(L, \mathcal{E}) \rightarrow(M, \mathcal{F}) .
$$

6.2. (Quasi-) Uniform structure on $\mathbf{C}(L, \mathcal{E})$. Entourages will allow us to construct the completion for uniform frames and quasi-uniform frames at once.

For a Cauchy map $\phi:(L, \mathcal{E}) \rightarrow M$ define $\phi \times \phi: L \oplus L \rightarrow M \oplus M$ by setting

$$
(\phi \times \phi)(E)=\bigvee\{\phi(a) \oplus \phi(b) \mid(a, b) \in E\} .
$$

We shall say that the Cauchy map $\phi$ is strict when its image generates $M$. The case of $\lambda_{(L, \mathcal{E})}$, where $M=\mathbf{C}(L, \mathcal{E})$, is obviously of this type.

6.2.1. Proposition. Let $\phi:(L, \mathcal{E}) \rightarrow M$ be a strict Cauchy map. If $\mathcal{E}$ is a quasi-uniformity (resp. uniformity), then

$$
\{(\phi \times \phi)(E) \mid E \in \mathcal{E}\}
$$

is a basis for a quasi-uniformity (resp. uniformity) $\mathcal{F}$ on $M$.

Proof. The fact that each $(\phi \times \phi)(E)$ is an entourage of $M$ follows from (C1). Condition (E2') follows easily from the fact that $\phi$ is a meet homomorphism and, in the symmetric case, (E4) is a consequence of the obvious formula $((\phi \times \phi)(E))^{-1}=(\phi \times \phi)\left(E^{-1}\right)$. In order to check condition (E3) it suffices to prove the following:

$$
F \circ F \leq E \Rightarrow(\phi \times \phi)(F) \circ(\phi \times \phi)(F) \leq(\phi \times \phi)(E) .
$$

This can be proved exactly as in (4.7.3) with $\phi$ in place of $j$ (since $\phi$ preserves binary meets and $\phi(0)=0$ ). 
Finally, the admissibility condition follows from (C2). Indeed, for each $b \in M, b=\bigvee_{i \in I} \phi\left(a_{i}\right)$ and therefore, by (C2),

$$
b=\bigvee_{i \in I} \bigvee\left\{\phi(x) \mid x \triangleleft_{\overline{\mathcal{E}}} a_{i}\right\}
$$

But, by $(*), E \circ(x \oplus x) \leq a_{i} \oplus a_{i}$ implies

$$
(\phi \times \phi)(E) \circ(\phi \times \phi)(x \oplus x) \leq(\phi \times \phi)\left(a_{i} \oplus a_{i}\right),
$$

that is, $(\phi \times \phi)(E) \circ(\phi(x) \oplus \phi(x)) \leq \phi\left(a_{i}\right) \oplus \phi\left(a_{i}\right)$. This shows that $x \triangleleft_{\overline{\mathcal{E}}} a_{i}$ implies $\phi(x) \triangleleft_{\overline{\mathcal{F}}} \phi\left(a_{i}\right) \leq b$. Hence,

$$
b=\bigvee_{i \in I} \bigvee\left\{\phi(x) \mid x \triangleleft_{\overline{\mathcal{E}}} a_{i}\right\} \leq \bigvee\left\{y \mid y \triangleleft_{\overline{\mathcal{F}}} b\right\} \leq b
$$

and $\overline{\mathcal{F}}$ is admissible.

The frame $\mathbf{C}(L, \mathcal{E})$ will be endowed with the quasi-uniformity (resp. uniformity) $\mathbf{C}(\mathcal{E})$ induced by $\mathcal{E}$ via $\lambda_{(L, \mathcal{E})}$, using the preceding result.

6.3. Theorem. Let $(L, \mathcal{E}),(M, \mathcal{F})$ be quasi-uniform (resp. uniform) locales. For every dense uniform embedding $j:(L, \mathcal{E}) \rightarrow(M, \mathcal{F})$ there is a dense quasi-uniform (resp. uniform) sublocale

$$
d: S(M, \mathcal{F}) \subseteq \mathbf{C}(L, \mathcal{E})
$$

and an isomorphism $\alpha:(M, \mathcal{F}) \cong S(M, \mathcal{F})$ such that $\lambda_{(L, \mathcal{E})}=d \alpha j$.

Proof. By 5.4 and 5.5 we have a frame homomorphism $h: \mathbf{C}(L, \mathcal{E}) \rightarrow M$ such that $h \lambda=j$. By 4.2, $M$ is join-generated by $L$. Hence we have, for an arbitrary $y \in M, y=\bigvee\{x \in L \mid x \leq y\}$ so that

$$
y=h(\bigvee\{\downarrow x \mid x \in L, x \leq y\},
$$

that is, $h$ is onto. Consequently the right adjoint $f=h_{*}$ is one-to-one, it is a dense embedding and we can set $S(M, \mathcal{F})=f[M]$ and endow it with the (quasi-) uniformity transferred from $M$.

6.3.1. Remark: The coframe of dense embeddings. Note that if two dense uniform embeddings $j_{i}:(L, \mathcal{E}) \rightarrow\left(M_{i}, \mathcal{F}_{i}\right)$ are equivalent, that is, if there is an isomorphism $\iota: M_{1} \rightarrow M_{2}$ such that $\iota j_{1}=j_{2}$, then $S\left(M_{1}, \mathcal{F}_{1}\right)=S\left(M_{2}, \mathcal{F}_{2}\right)$. Furthermore, there is the smallest dense uniform embedding of $(L, \mathcal{E})$, namely the identity, and each embedding $\lambda[L] \subseteq S$ with $S$ a sublocale of $\mathbf{C}(L, \mathcal{E})$ is dense. Thus, the construction $S(-)$ provides a one-to-one correspondence between the system of all (equivalence classes of) uniform embeddings $j:(L, \mathcal{E}) \rightarrow(M, \mathcal{F})$ and the lattice of all sublocales of $\mathbf{C}(L, \mathcal{E})$ containing $\lambda[L]$.

Consequently, the system of all (equivalence classes of) uniform dense embeddings of a fixed uniform frame $(L, \mathcal{E})$ naturally constitutes a subcoframe of $\mathbf{C}(L, \mathcal{E})$. 
To have the statement quite correct, we have to complete the theorem above by the following simple observation that shows that the sublocale $S(M, \mathcal{F})$ is uniquely determined. Consider the following diagram in Loc

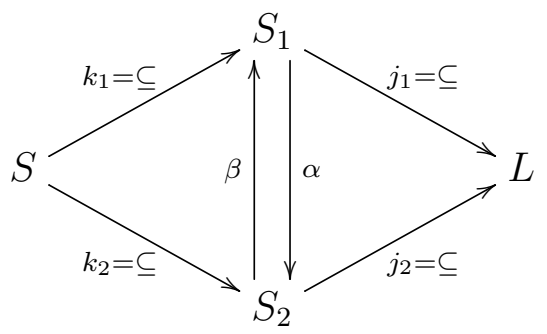

with $j_{i}, k_{i}$ dense embeddings, $\alpha, \beta$ mutually inverse isomorphisms, $k_{2} \alpha=$ $k_{1}$, and $k_{1} \beta=k_{2}$. Then $j_{1} \beta k_{2}=j_{1} k_{1}=j_{2} k_{2}$ and since $k_{2}$, being dense, is an epimorphism in Loc, $j_{1} \beta=j_{2}$ is an actual embedding, we have $\beta$ an actual embedding, and similarly for $\alpha$; hence $\alpha, \beta$ are identities, and $S_{1}=S_{2}$.

6.4. Proposition. Let $(L, \mathcal{E})$ be a quasi-uniform frame, resp. a uniform frame. In each case, $\mathbf{C}(L, \mathcal{E})$ is complete, and hence a completion of $(L, \mathcal{E})$.

Proof. Let $k: \mathbf{C}(L, \mathcal{E}) \rightarrow(M, \mathcal{F})$ be a dense uniform embedding. Consider the diagram

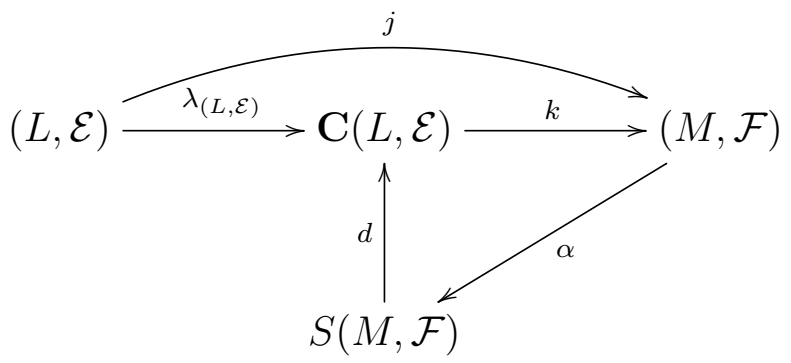

with $j=k \lambda_{(L, \mathcal{E})}$ and $\alpha$ and $d$ given by 6.3. Then $\lambda=\lambda_{(L, \mathcal{E})}=d \alpha j=$ $d \alpha k \lambda$. Since $\lambda$ and $k$ are epimorphisms, this yields $d \alpha k=\operatorname{id}_{\mathbf{C}(L, \mathcal{E})}$ which makes $k$ an isomorphism.

6.5. Theorem. Let $(L, \mathcal{E})$ be a quasi-uniform frame, resp. a uniform frame. Then $(L, \mathcal{E})$ is complete iff each Cauchy map $(L, \mathcal{E}) \rightarrow M$ is a frame homomorphism.

Proof. If $(L, \mathcal{E})$ is complete then $\lambda$ is an isomorphism, and hence we have $\phi=h \lambda$ a homomorphism. On the other hand, if each Cauchy map $(L, \mathcal{E}) \rightarrow M$ is a frame homomorphism, then in particular $\lambda:(L, \mathcal{E}) \rightarrow$ 
$\mathrm{C}(L, \mathcal{E})$ is one. For the $v=v_{(L, \mathcal{E})}$ we have $v \lambda=\mathrm{id}$ and since $v$, being dense, is a monomorphism, we also have $\lambda v=\mathrm{id}$, and $\lambda$ is an isomorphism.

6.5.1. Remark. The cover uniformity analogue of the symmetric case in the previous result is due to B. Banaschewski $([2,3])$.

6.5.2. Example. Let $L=(L, \mathcal{E})$ be a complete uniform frame which is not Boolean, and let $B=\mathfrak{B}(L, \mathcal{E})$ be its Booleanization endowed with the uniformity induced by $\mathcal{E}$. Since the right adjoint of the frame homomorphism $h=\left(a \mapsto a^{* *}\right): L \rightarrow B$ is a uniform dense embedding and not an isomorphism, $B$ is not complete. Hence there exists a Cauchy map $\phi: B \rightarrow M$ which is not a homomorphism. Then $\phi \cdot h$ is not Cauchy, because it is not a homomorphism ( $L$ is complete) - if it were, $\phi$ would be one as well, as $h$ is onto.

6.6. Lemma. Let $(L, \mathcal{E})$ be a quasi-uniform frame. For a weakly Cauchy map $\phi:(L, \mathcal{E}) \rightarrow M$ define

$$
\phi^{\circ}:(L, \mathcal{E}) \rightarrow M
$$

by setting $\phi^{\circ}(a)=\bigvee\left\{\phi(b) \mid b \triangleleft_{\overline{\mathcal{E}}} a\right\}$. Then $\phi^{\circ}$ is a Cauchy map.

Proof. If $E \in \mathcal{E}$ consider the $E^{\triangleleft}$ from 3.4. Then

$$
\bigvee\left\{\phi^{\circ}(a) \mid(a, a) \in E\right\}=\bigvee\left\{\phi(b) \mid b \triangleleft_{\overline{\mathcal{E}}} a,(a, a) \in E\right\}
$$

and, using the property in 3.3,

$$
\bigvee\left\{\phi(b) \mid b \triangleleft_{\overline{\mathcal{E}}} a,(a, a) \in E\right\} \geq \bigvee\left\{\phi(b) \mid(b, b) \in E^{\triangleleft}\right\}=1
$$

(indeed, for any $(b, b) \in E^{\triangleleft}$ with $b \triangleleft_{\mathcal{E}}^{1} x, b \triangleleft_{\mathcal{E}}^{2} y$ and $(x, y) \in E, 3.3$ yields $b \triangleleft_{\overline{\mathcal{E}}} x \wedge y$ and, of course, $\left.(x \wedge y, x \wedge y) \in E\right)$.

Next,

$\bigvee\left\{\phi^{\circ}(b) \mid b \triangleleft_{\overline{\mathcal{E}}} a\right\}=\bigvee\left\{\phi(c) \mid c \triangleleft_{\overline{\mathcal{E}}} b \triangleleft_{\overline{\mathcal{E}}} a\right\}=\bigvee\left\{\phi(c) \mid c \triangleleft_{\overline{\mathcal{E}}} a\right\}=\phi^{\circ}(a)$ since $\triangleleft_{\overline{\mathcal{E}}}$ interpolates.

In particular, when $(L, \mathcal{E})$ is a uniform frame, the Cauchy map $\phi^{\circ}$ is given by

$$
\phi^{\circ}(a)=\bigvee\left\{\phi(b) \mid b \triangleleft_{\mathcal{E}} a\right\} .
$$

6.7. Theorem. The completion $\mathbf{C}$ is functorial in both, the symmetric and the non-symmetric, cases.

Proof. For a uniform frame homomorphism $h:(L, \mathcal{E}) \rightarrow(M, \mathcal{F})$ consider the weak Cauchy map $\lambda \cdot h:(L, \mathcal{E}) \rightarrow \mathbf{C}(M, \mathcal{F})$ and the Cauchy map $(\lambda \cdot h)^{\circ}:(L, \mathcal{E}) \rightarrow \mathbf{C}(M, \mathcal{F})$. Then, we have the frame homomorphism $g: \mathbf{C}(L, \mathcal{E}) \rightarrow \mathbf{C}(M, \mathcal{F})$ defined by

$$
g(U)=\bigvee\left\{(\lambda h)^{\circ}(u) \mid u \in U\right\}=\bigvee\left\{\downarrow h(x) \mid x \triangleleft_{\overline{\mathcal{E}}} u \in U\right\} .
$$


Denoting this $g$ by $\mathbf{C}(h)$ we obtain that

$$
\begin{aligned}
v_{(M, \mathcal{F})} \cdot \mathbf{C}(h) & =\bigvee\left\{v(\downarrow h(x)) \mid x \triangleleft_{\overline{\mathcal{E}}} u \in U\right\}= \\
& =\bigvee\left\{h(x) \mid x \triangleleft_{\overline{\mathcal{E}}} u \in U\right\}= \\
& =\bigvee\{h(u) \mid u \in U\}= \\
& =h(\bigvee\{u \mid u \in U\})=h \cdot v_{(L, \mathcal{E})} .
\end{aligned}
$$

Since the homomorphisms $v_{(L, \mathcal{E})}$ are dense and hence monomorphic in our category, it immediately follows that $\mathbf{C}\left(h_{1} h_{2}\right)=\mathbf{C}\left(h_{1}\right) \mathbf{C}\left(h_{2}\right)$.

Checking that $\mathbf{C}(h)=g$ is uniform is straightforward.

\section{MORE ON THE ASYMMETRIC CASE}

7.1. Let $(L, \mathcal{E})$ be a quasi-uniform frame. Consider the associated uniformity $\overline{\mathcal{E}}$ on $L$ and the corresponding completion

$$
\lambda=\lambda_{(L, \overline{\mathcal{E}})}:(L, \overline{\mathcal{E}}) \rightarrow(\mathbf{C}(L, \overline{\mathcal{E}}), \mathbf{C}(\overline{\mathcal{E}})) .
$$

By 6.2.1,

$$
\{(\lambda \times \lambda)(E) \mid E \in \mathcal{E}\}
$$

forms a basis for a quasi-uniformity $\mathbf{C}(\mathcal{E})$ on $\mathbf{C}(L, \overline{\mathcal{E}})$ and thus we have a quasi-uniform frame

$$
(\mathbf{C}(L, \overline{\mathcal{E}}), \mathbf{C}(\mathcal{E}))
$$

also defined in the sublocale $\mathbf{C}(L, \overline{\mathcal{E}})$ of $\mathfrak{D} L$.

\subsubsection{Proposition. $\overline{\mathrm{C}(\mathcal{E})}=\mathrm{C}(\overline{\mathcal{E}})$.}

Proof. $\overline{\mathbf{C}(\mathcal{E})} \subseteq \mathbf{C}(\overline{\mathcal{E}})$ follows from the fact that $\mathbf{C}(\overline{\mathcal{E}})$ is a uniformity and $\mathbf{C}(\mathcal{E}) \subseteq \mathbf{C}(\overline{\mathcal{E}})$ (since $\{(\lambda \times \lambda)(E) \mid E \in \overline{\mathcal{E}}\}$ is a basis of $\mathbf{C}(\overline{\mathcal{E}})$, by $4.5)$.

On the other hand, by the characterization in 4.5 ,

$$
\lambda:(L, \mathcal{E}) \rightarrow((\mathbf{C}(L, \overline{\mathcal{E}}), \mathbf{C}(\mathcal{E}))
$$

is clearly a dense uniform embedding. Hence, by 4.7.1,

$$
\lambda:(L, \overline{\mathcal{E}}) \rightarrow(\mathbf{C}(L, \overline{\mathcal{E}}), \overline{\mathbf{C}(\mathcal{E})})
$$

is a dense uniform embedding. Since $(\mathbf{C}(L, \overline{\mathcal{E}}), \mathbf{C}(\overline{\mathcal{E}}))$ is the completion of $(L, \overline{\mathcal{E}})$, this implies immediately that $\mathbf{C}(\overline{\mathcal{E}})=\overline{\mathbf{C}(\mathcal{E})}$.

7.2. Proposition. Let $(L, \mathcal{E})$ be a quasi-uniform frame. Then $(L, \mathcal{E})$ is complete iff it is bicomplete (i.e. $(L, \overline{\mathcal{E}})$ is complete). 
Proof. $\Leftarrow$ : Let $j:(L, \mathcal{E}) \rightarrow(M, \mathcal{F})$ be a dense uniform embedding. By 4.7.1, $j:(L, \overline{\mathcal{E}}) \rightarrow(M, \overline{\mathcal{F}})$ is a dense uniform embedding and thus it is an isomorphism.

$\Rightarrow$ : Let $j:(L, \overline{\mathcal{E}}) \rightarrow(M, \mathcal{F})$ be a dense uniform embedding. By 4.7.2, $\{(j \times j)(E) \mid E \in \mathcal{E}\}$ is a basis for a quasi-uniformity $\mathcal{G}$ in $M$, and by $4.5, j:(L, \mathcal{E}) \rightarrow(M, \mathcal{G})$ is a dense uniform embedding hence an isomorphism.

7.3. Theorem. $(\mathbf{C}(L, \overline{\mathcal{E}}), \mathbf{C}(\mathcal{E}))$ is the completion of $(L, \mathcal{E})$.

Proof. $(\mathbf{C}(L, \overline{\mathcal{E}}), \mathbf{C}(\mathcal{E}))$ is complete by the two preceding propositions and

$$
\lambda:(L, \mathcal{E}) \rightarrow((\mathbf{C}(L, \overline{\mathcal{E}}), \mathbf{C}(\mathcal{E}))
$$

is a dense uniform embedding by 4.5 .

7.4. Corollary. If the given $(L, \mathcal{E})$ is a uniform frame, then the quasiuniform completion $(\mathbf{C}(L, \overline{\mathcal{E}}), \mathbf{C}(\mathcal{E}))$ is the uniform completion of $(L, \mathcal{E})$.

\section{ACKNOWLEDGEMENTS}

We are most grateful to the referee for all valuable comments and suggestions that improved the paper and, in particular, for alerting us for a mistake in the previous version of 6.2.1.

Further, we acknowledge support from grants P202/12/G061 (Grant Agency of the Czech Republic) and MTM2015-63608-P (Ministry of Economy and Competitiveness of Spain) and from the Centre for Mathematics of the University of Coimbra (UID/MAT/00324/2013 funded by FCT/MCTES and FEDER through the Partnership Agreement PT2020).

\section{REFERENCES}

[1] R. N. Ball and A. Pultr, Extending semilattices to frames using sites and coverages, Math. Slovaca 64 (2014) 527-544.

[2] B. Banaschewski, Completion in Pointfree Topology, Lecture Notes in Mathematics, University of Cape Town, 1996.

[3] B. Banaschewski, Uniform completion in pointfree topology, in: Topological and Algebraic Structures in Fuzzy Sets, pp. 19-56. Trends Log. Stud. Log. Libr., vol. 20, Kluwer Academic Publishers, Boston, Dordrecht, London, 2003.

[4] B. Banaschewski, S.S. Hong and A. Pultr, On the completion of nearness frames, Quaest. Math. 21 (1998) 19-37.

[5] B. Banaschewski, G. C. L. Brümmer and K. A. Hardie, Biframes and bispaces, Quaest. Math. 6 (1983) 13-25.

[6] B. Banaschewski and A. Pultr, Samuel compactification and completion of uniform frames, Math. Proc. Cambridge Phil. Soc. 108 (1990) 63-78. 
[7] B. Banaschewski and A. Pultr, Cauchy points of uniform and nearness frames, Quaest. Math. 19 (1996) 101-127.

[8] B. Banaschewski and A. Pultr, Completion and Samuel compactification of nearness and uniform frames, Portug. Math. 69 (2012) 113-126.

[9] C. H. Dowker and D. Strauss, Sums in the category of frames, Houston J. Math. 3 (1977), 7-15.

[10] P. Fletcher and W.F. Lindgren, Quasi-Uniform Spaces, Marcel Dekker, New York, 1982.

[11] J. Frith and W. Hunsaker, Completion of quasi-uniform frames, Appl. Categ. Struct. 7 (1999) 261-270.

[12] J. Frith, W. Hunsaker and J. Walters-Wayland, The Samuel compactification of a quasi-uniform frame, Topology Proc. 23 (1998) 115-126.

[13] S. S. Hong and Y. K. Kim, Cauchy completions of nearness frames, Appl. Categ. Struct. 3 (1995) 371-377.

[14] J. R. Isbell, Atomless parts of spaces, Math. Scand. 31 (1972) 5-32.

[15] P. T. Johnstone, Stone Spaces, Cambridge Niversity Press, Cambridge 1982.

[16] Y.K. Kim, Cauchy completions of quasi-uniform frames, Kyungpook Math. J. 38 (1998) 429-437.

[17] I. Kříž, A direct description of uniform completion in locales and a characterization of LT-groups Cahiers Topologie Géom. Différentielle Catég., 27 (1986) 19-34.

[18] W. F. Lindgren and P. Fletcher, A construction of the pair completion of a quasi-uniform space, Canad. Math. Bull. 21 (1978) 53-59.

[19] J. Picado, Weil uniformities for frames, Comment. Math. Univ. Carolinae 36 (1995) 357-370.

[20] J. Picado, Frame quasi-uniformities by entourages, in: Symposium on Categorical Topology, pp. 161-175, Univ. Cape Town, Rondebosch, 1999.

[21] J. Picado, Structured frames by Weil entourages, Appl. Categ. Struct. 8 (2000) 351-366.

[22] J. Picado and A. Pultr, Frames and locales: Topology without points, Frontiers in Mathematics, vol. 28, Springer, Basel (2012).

[23] J. Picado and A. Pultr, Entourages, covers and localic groups, Appl. Categ. Struct. 21 (2013) 49-66.

[24] J. Picado and A. Pultr, Notes on the product of locales, Math. Slovaca 65 (2015) 247-264.

[25] A. Pultr and A. Tozzi, Completion and coproducts of nearness frames, in: Symposium on Categorical Topology, pp. 177-186, Univ. Cape Town, Rondebosch, 1999.

CMUC, Department of Mathematics, University of Coimbra, 3001-501 CoImBra, PORTUGAL

Email address: picado@mat.uc.pt

Department of Applied Mathematics and iti, MFF, Charles UniverSity, Malostranské nám. 24, 11800 Praha 1, Czech Republic

Email address: pultr@kam.mff.cuni.cz 